\title{
Multi-Paradigm Modelling of Cyber-Physical Systems
}

\author{
Extended Abstract \\ Hans Vangheluwe \\ University of Antwerp/Flanders Make \\ Antwerp, Belgium \\ Hans.Vangheluwe@uantwerpen.be
}

\begin{abstract}
The networking of multi-physics (mechanical, electical, hydraulic, biochemical, ...) with computational systems (control systems, signal processing, logical inferencing, planning, ...) processes, interacting with often uncertain environments, with human actors, in a socio-economic context, leads to so-called Cyber-Physical Systems (CPS).

Cyber-physical systems are reaching a hitherto unseen level of complexity. To date, no unifying theory nor systematic design methods, techniques and tools exist for such systems. Individual (mechanical, electrical, network or software) engineering disciplines only offer partial solutions.

Multi-paradigm Modelling (MPM) proposes to model every part and aspect of such complex systems explicitly, at the most appropriate level(s) of abstraction, using the most appropriate modelling formalism(s). This includes the explicit modelling of the often complex engineering workflows. Modelling language engineering, including model transformation languages, and the study of the semantics of these languages, are used to realize MPM. MPM is seen as an effective answer to the challenges of designing CPS.

This presentation introduces some of the challenges of collaborative development of CPS as well as possible multi-paradigm modelling solutions such as (in-)consistency management and cosimulation.
\end{abstract}

\section{CCS CONCEPTS}

- General and reference $\rightarrow$ Design; • Computer systems organization $\rightarrow$ Embedded and cyber-physical systems;

\section{KEYWORDS}

Multi-Paradigm Modelling (MPM), Cyber-Physical Systems (CPS), Simulation

\section{ACM Reference Format:}

Hans Vangheluwe. 2018. Multi-Paradigm Modelling of Cyber-Physical Systems: Extended Abstract. In SEsCPS'18: SEsCPS'18:IEEE/ACM 4th International Workshop on Software Engineering for Smart Cyber-Physical Systems, May 27-fune 3, 2018, Gothenburg, Sweden. ACM, New York, NY, USA, Article 4, 1 page. https://doi.org/10.1145/3196478.3196479

Permission to make digital or hard copies of part or all of this work for personal or classroom use is granted without fee provided that copies are not made or distributed for profit or commercial advantage and that copies bear this notice and the full citation on the first page. Copyrights for third-party components of this work must be honored For all other uses, contact the owner/author(s).

SEsCPS'18, May 27-June 3, 2018, Gothenburg, Sweden

() 2018 Copyright held by the owner/author(s)

ACM ISBN 978-1-4503-5728-9/18/05 ..\$15.00

https://doi.org/10.1145/3196478.3196479

\section{BIOGRAPHY}

Hans Vangheluwe is a Professor in the Antwerp Systems and Software Modelling (AnSyMo) group within the Department of Mathematics and Computer Science at the University of Antwerp in Belgium, where he is a founding member of the NEXOR IOF Consortium on Cyber-Physical Systems (CPS). He is an Adjunct Professor in the School of Computer Science at McGill University, Montréal, Canada. AnSyMo is an Core Research Lab of Flanders Make, the strategic research centre for the Flemish manufacturing industry. He heads the Modelling, Simulation and Design Lab (MSDL), distributed over the University of Antwerp and McGill.

In a variety of projects, often with industrial partners, he develops and applies the model-based theory and techniques of MultiParadigm Modelling (MPM) in diverse application domains. He is the chair of the EU COST Action IC1404 Multi-Paradigm Modelling for Cyber-Physical Systems (MPM4CPS). 